\title{
A new damage detection method: Big Bang-Big Crunch (BB-BC) algorithm
}

\author{
Zahra Tabrizian $^{\mathrm{a}}$, Ehsan Afshari ${ }^{\mathrm{b}}$, Gholamreza Ghodrati Amiri ${ }^{\mathrm{c}, *}$, Morteza Hossein Ali Beigy ${ }^{\mathrm{a}}$ and \\ Seyed Mohammad Pourhoseini Nejad ${ }^{\mathrm{d}}$ \\ ${ }^{a}$ College of Civil Engineering, Babol Noshirvani University of Technology, Babol, Iran \\ ${ }^{\mathrm{b}}$ School of Civil Engineering, Iran University of Science and Technology, Narmak, Tehran, Iran \\ ${ }^{\mathrm{C}}$ Centre of Excellence for Fundamental Studies in Structural Engineering, Iran University of Science and \\ Technology, Narmak, Tehran, Iran \\ ${ }^{\mathrm{d}}$ Department of Civil Engineering, Yazd University, Yazd, Iran
}

Received 15 August 2012

Revised 30 October 2012

Accepted 14 January 2013

\begin{abstract}
The present paper aims to explore damage assessment methodology based on the changes in dynamic parameters properties of vibration of a structural system. The finite-element model is used to apply at an element level. Reduction of the element stiffness is considered for structural damage. A procedure for locating and quantifying damaged areas of the structure based on the innovative Big Bang-Big Crunch (BB-BC) optimization method is developed for continuous variable optimization. For verifying the method a number of damage scenarios for simulated structures have been considered. For the purpose of damage location and severity assessment the approach is applied in three examples by using complete and incomplete modal data. The effect of noise on the accuracy of the results is investigated in some cases.

A great unbraced frame with a lot of damaged element is considered to prove the ability of proposed method. More over BB$\mathrm{BC}$ optimization method in damage detection is compared with particle swarm optimizer with passive congregation (PSOPC) algorithm.

This work shows that BB-BC optimization method is a feasible methodology to detect damage location and severity while introducing numerous advantages compared to referred method.
\end{abstract}

Keywords: Structural damage detection, finite-element model, dynamic parameters properties, Big Bang-Big Crunch algorithm

\section{Introduction}

During a structure life time, it may suffer large damage without showing any external indications of damage. If the damage could not be detected in time, the current damage may specify by a sudden failure or a fatal disaster of structure, which causes human life and property damages. Therefore, one essential step in the structural health monitoring is to develop a methodology for the accurate and reliable assessment of structural damage. Besides it is very important to ensure the integrity, stability of structures and damage location, to prevent extensive failure, and to reduce the cost of maintenance $[1,2]$.

In this paper the method that has been used is either visual or nondestructive and gives the best answers. In view of the relative unreliability of visual inspections [3], it is generally acknowledged that more objective means

\footnotetext{
${ }^{*}$ Corresponding author: Gholamreza Ghodrati Amiri, Centre of Excellence for Fundamental Studies in Structural Engineering, Iran University of Science and Technology, Narmak, Tehran, Iran. Tel.: +98 217724 0399; Fax: +98 217724 0398; E-mail: Ghodrati@iust.ac.ir.
} 
for assessing the structural health of bridges are required. Such means could include local nondestructive evaluation methods [4,5] or global methods, which use changes in the overall response of a structure as indicators of damage [68].

Global damage detection methods applicable to complex structures, techniques based on modal testing and signal processing constitution for damage identification in civil and mechanical engineering has been considered by Ewins [9]. Doebling et al. [10]; Hu et al. [11] studied and reviewed the methods works with changes in the dynamic characteristics of the structure, such as natural frequencies and mode shapes, etc. Identification of the location and severity of damage was used to compare the damaged and healthy structure. Generally some dynamic parameters such as natural frequencies and mode shapes are damage indicators. Because the parameter can be determined by measuring at only one point of the structure the methods based on the measurement of natural frequencies are very simple Salawu [12]; Bicanic et al. [13]. Since some derivatives of mode shapes, such as mode shape curvatures, are more sensitive to small perturbations than modal displacements, therefore is used to damage detection Pandey et al. [14]. Model updating methods based on traditional optimization techniques was studied by Mottershead and Friswell 1993. This New methods have been developed in inverse procedures which called evolutionary algorithms [15].

In many structures may occur some local damage during their functional age .In order to develop the efficiency of the structures, it is necessary to properly identify the damage places and their severity, and then repair them. There are many methods that have been introduced correctly find out the place and severity of structural damage.

The genetic algorithms (GAs) as one of the most important artificial intelligence algorithms, it can solve a global minimum theoretically without utilizing the gradient information [16]. GAs are employed by many researchers for structural damage detection based on model-up dating methods [17]. Some researchers studied a nondestructive global damage detection and assessment methodology based on the changes in frequencies and mode shapes of vibration of a structural system. It was shown that the proposed GA yields a suitable damage places and severity detection from traditional methods [18]. BP-CGA damage detection method proposed by Gerist et al. [19]; that is the combination of basis pursuit and continuous genetic algorithm and is relatively accurate and fast.

In addition the GA method, the particle swarm optimization (PSO) and ant colony optimization (ACO) methods, two biologically inspired algorithms, are used for the purpose of structural healthy monitoring following the modelupdating approach [20,21].

For determining place and severity of multiple structural damage by combining the adaptive neuro-fuzzy inference system (ANFIS) and particle swarm optimization (PSO) a two-stage method has been studied by Fallahian and Seyedpoor [22]. In other study, it was determined the place and severity of multiple damage by iteratively searching for a combination of structural responses that maximizes a correlation coefficient named the multiple damage location assurance criterion (MDLAC) by the use of a genetic algorithm (GA) [23]. A Hybrid technique was used for damage detection by including of a real-parameter genetic algorithm and grey relation analysis [24]. First the researchers used a grey relation analysis to keep out impossible damage places which help to reduce the number of design variables. Second, a real-parameter genetic algorithm was applied for finding the actual damage. A two-stage method was utilized to determine the place and severity of structural damage via an information fusion technique and a GA [25]. In other research two stage methods was used to identify the place and severity of damage in a bridge. First, two objective functions are defined to minimize the sum of differences between the modal data before and after damage in classic method and other objective functions defined derived from modal flexibility. Secondly, an improved particle swarm optimization (PSO) algorithm is developed to solve the multiple-objective optimization problem on bridge damage detection. The results show that the locating and quantifying damaged elements of bridge structures was detected relevantly [26].

Kourehli et al. Showed an effective method for damage detection and estimation in structures based on incomplete modal data of a damaged structure via a pattern search algorithm. The objective function based on the condensed mass and stiffness Matrices was formulated [27].

An immunity enhanced particle swarm optimization (IEPSO) algorithm, which combines particle swarm optimization (PSO) with the artificial immune system, is proposed for damage detection of beam and truss structures by Fei Kang. The objective function for damage detection is based on vibration data, such as natural frequencies and mode shapes. The feasibility and efficiency of IEPSO are compared with the basic PSO. Results show that this method is efficient on determining the place and the extents of structure damages [28]. 
A simple methodology to determine the location and amount of crack in beam like structures based on the incremental particle swarm optimization technique is presented by Nanda et al. A comparison is made for assessing the performance of standard particle swarm optimization and the incremental particle swarm optimization technique for detecting crack in structural members. Finally simulated results demonstrate that the developed method is capable of detecting and estimating the extent of damages with sufficient accuracy [29].

Imperialist competitive algorithm was used for detection and estimation of structural damage on the basis of modal parameters of a damaged structure. The performance of the this method has been verified through using a benchmark problem provided by the IASC-ASCE Task Group on Structural Health Monitoring and a number of numerical examples. The results clearly showed the superiority of this method in comparison with energy index method [30].

In the present work, a new damage identification method namely Big Bang-Big Crunch (BB-BC) algorithm is proposed to predict the damage place and severity for different types of structures. The structure is modeled with the finite-element method and the damage identification is then carried out at the element with complete and incomplete modal data. The effect of noise in results of the BB-BC algorithm is considered in some cases by assigning the noise in natural frequencies. The graphs indicate that the noise may reduce the accuracy of answers slightly, especially in the case of incomplete modal data.

To demonstrate the effectiveness of this approach, three numerical example analyses on beam, truss bridge and plane frame are presented. Finally, the power of BB-BC algorithm in damage detection is compared with particle swarm optimizer with passive congregation algorithm (PSOPC). It is concluded that using this algorithm, good results of damage detection can obtain with low computational cost.

\section{Theoretical background}

In a specific classification structural damage detection techniques can be divided in dynamic or static methods [31]. From the finite element modeling point of view, the structural global stiffness matrix is assembled from elemental stiffness. Structural damage can reduce the stiffness and increase the flexibility of structures. Mathematically, damage influences the global stiffness typically. Physically, damage causes changes in structural dynamic properties, such as natural mode shapes and frequencies.

The natural frequencies of a structure can be considered to be important with the dynamic data. Determining the level of correlation between the calculated and predicted natural frequencies can present a simple tool for finding the place and severity of structural damage [32]. Structural damage detection is calculated with changes in structural characteristics because of damage occurrence. Vibration-based techniques have discussed by Doebling in a literature review [33]. In this part the construction of dynamics of damaged structures is presented.

For evaluating the correlation coefficients, the parameter vector is used that consists of the ratios of the first $n_{f}$ natural frequency changes $\Delta F$ due to structural damage:

$$
\Delta F=\frac{F_{h}-F_{d}}{F_{h}}
$$

Where $F_{h}$ and $F_{d}$ denote the natural frequency vectors of the healthy and damaged structure. Similarly, the parameter vector predicted from an analytical model can be defined correspondingly as

$$
\delta F(X)=\frac{F_{h}-F(X)}{F_{h}}
$$

Where $F(X)$ is a natural frequency vector that can be predicted from an analytic model and $X=\left[x^{1}, x^{2}, \ldots, x^{n}\right]$ represents a damage variable vector containing the damage severity of all $n$ structural elements.

It is noted that, if the natural frequency vector of undamaged structure (before the injury) is not available, can be determined by using dynamic analysis. So, for use the above relationships, it is required to having dynamic data of undamaged structure. 
Given a pair of parameter vectors, one can estimate the level of correlation in several ways. An efficient way is to evaluate a correlation-based index, termed the multiples damage location assurance criterion (MDLAC), and covered in the following form [32]:

$$
\operatorname{MDLAC}(X)=\frac{\left|\Delta F^{T} \cdot \delta F(X)\right|^{2}}{\left(\Delta F^{T} \cdot \Delta F\right)\left(\delta F^{T}(X) \cdot \delta F(X)\right)} \quad \text { and } 0<M D L A C<1
$$

Two frequency change vectors are compared with MDLAC that one calculated from the structural tests and the other from a structural model analysis. When the vector of analytical frequencies becomes the same to the frequency vector of the damaged structure, MDLAC will be maximal, That is $F(X)=F_{d}$. So considering this theory can be used to find a set of damage variables maximizing the MDLAC using an optimization algorithm:

$$
\begin{array}{ll}
\text { Find } & X=\left[x_{i}, x_{2}, \ldots, x_{n}\right] \\
\text { Maximize: } & w(X)=\operatorname{MDLAC}(X) \\
& x_{i} \in\left[\begin{array}{ll}
0 & 1
\end{array}\right]
\end{array}
$$

The damage severity can take values only from the set that given from [0 1], set of continuous values. Moreover, the objective function that should be maximized is $w$. As mentioned the damage occurrence in a structural element, decreases the element stiffness. Thus, one of the methods for the damage identification problem, is simulation damage by decreasing one of the stiffness parameters of the element such as the modulus of elasticity $(E)$, crosssectional area $(A)$, inertia moment $(I)$, and .... In this study, the damage variables are defined via a relative reduction of the elasticity modulus of an element as

$$
\begin{aligned}
K_{i}^{d} & =\left(1-x_{i}\right) K_{i}^{h} \\
K_{i}^{d} & =\text { stiffness matrix of damaged element } i \\
K_{i}^{h} & =\text { stiffness matrix of healthy element } i
\end{aligned}
$$

The MDLAC as an objective function for the optimization algorithm is more sensitive to damaged elements than undamaged elements. It means, this method can find the true place of the damaged elements but it may find an undamaged element as a damaged one. Therefore, in this study a new function is discussed, a new function is presented as below [34];

$$
\operatorname{obj}(X)=\frac{1}{n_{f}} \sum_{i=1}^{n_{f}} \frac{\min \left(f_{x i}, f_{d i}\right)}{\max \left(f_{x i}, f_{d i}\right)}
$$

Where $f_{x i}$ and $f_{d i}$ are the $i$ th components of vectors $F(X)$ and $F_{d}$, correspondingly.

The $\operatorname{obj}(X)$ function can rapidly find the locations of healthy elements when compared to the MDLAC; however, it is very probable that it finds a damaged element as a healthy one. Therefore, in this study, a combinational function of Eqs (3) and (6), called here the efficient correlation-based index (ECBI), is used as [34]

$$
\operatorname{ECBI}(X)=\frac{1}{2}(\operatorname{MDLAC}(X)+\operatorname{obj}(X))
$$

To detect damage location, because of the better knowledge variations in the locality, it has an effect on area mode shapes offer a better option (Salane and Baldwin [35]; Salawu and Williams [36]; Ndambi et al. [37]). Besides the accuracy of this approach depends on the quality and place selection of the measurements in experimental test which effect to identify the damage. Whereas, only a few natural frequencies and mode shapes of the lower modes are available to assume, frequency objective functions from incomplete data are considered in this paper to detect damage. It helps to detect damage in absence of complete data. 


\subsection{Big Bang Big-Crunch theory}

A new optimization algorithm namely the Big Bang-Big Crunch (BB-BC) is developed by Erol and Eksin [38]. This method is based on one of the evolutionary theories of the universe namely, the Big Bang and Big Crunch theory, which has low computational cost and a high convergence speed. This algorithm consists of two steps: The Big Bang where a random disordered state of candidate are produced within the allowable space, followed by Big Crunch, where candidates are averaged based on their quality and put into an order. In each step of BB-BC algorithm the new population is generated based on information obtained from the previous step, in other hand, the position of each new candidate solution is formed around the center of mass that is calculated from the Big Crunch stage. After a number of sequential Big Bangs and Big Crunches, where the distribution of randomness within the search space during the Big Bang becomes smaller and smaller about the average point computed during the Big Crunch, the algorithm converges to a solution.

\subsection{A Big Bang-Big Crunch algorithm}

This method is similar to the GA in respect to creating an initial population randomly. The creation of the initial population randomly is called the Big Bang. In this step, the candidate solutions are spread all over the search space in an uniform manner.

If there is a limitation for each component of population and any of the components exceeded the boundaries, it is necessary to transfer these values to the adjacent boundaries. The Big Bang is followed by the Big Crunch. The Big Crunch is a convergence operator that has many inputs but only one output, which can be named as the center of 'mass', since the only output has been derived by calculating the center of mass. Here, the term mass refers to the inverse of the fitness function value. The point representing the center of mass that is denoted by $x^{c}$ is calculated according to [38]:

$$
\vec{x}^{c}=\frac{\sum_{i=1}^{N} \frac{1}{f^{i}} \vec{x}^{i}}{\sum_{i=1}^{N} \frac{1}{f^{i}}}
$$

Where $x^{i}$ is a point within an $n$-dimensional search space generated; $f^{i}$ is a fitness function value of this point, and $N$ is the population size of algorithm in Big Bang step.

After the Big Crunch, the algorithm must create new members to be used as the Big Bang of the next iteration [38]:

$$
x_{i}^{\text {new }}=X^{c}+\sigma
$$

Where $\sigma$ is calculated using following equation:

$$
\sigma=\frac{r \alpha\left(x_{\max }-x_{\min }\right)}{k}
$$

$\alpha$ is the parameter limiting the size of the search space; $r$ is a random number from a standard normal distribution which changes for each candidate; $x_{\max }$ and $x_{\min }$ are the upper and lower limits on the values of the optimization problem variables, and $k$ is the number of Big Bang iterations. After the second explosion, the center of mass is recalculated. These successive explosion and contraction steps are carried repeatedly until a stopping criterion has been met.

Two additional steps implemented to improve computational efficiency and performance [39]. First, positions of candidate solutions at the beginning of each Big Bang are normally distributed around a new point located between the center of mass, $X^{c}$, and the best global solution, $X^{\text {gbest }}$, using the following:

$$
X^{(k+1, i)}=\beta X^{c(k)}+(1-\beta) X^{\text {gbest }(k)}+\sigma
$$

Where $\beta$ is the parameter for controlling the influence of the $X^{\text {gbest }(k)}$ on the location of new candidate solutions; $X^{\text {gbest }(k)}$ is the position of the best global solution. Numerical studies indicate that there is significant improvement 


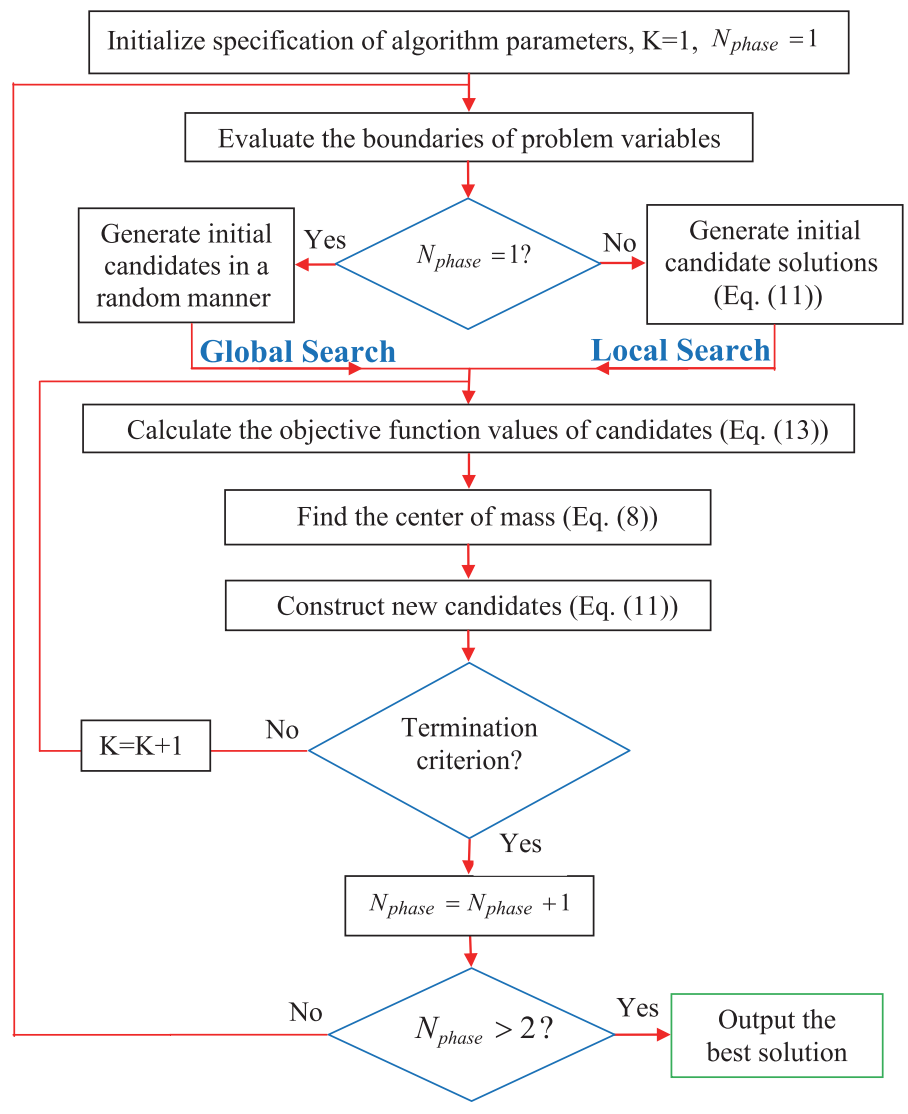

Fig. 1. Flowchart of the BB-BC algorithm.

in the quality of the solutions and the computational efficiency of the BB-BC algorithm using Eq. (11) over the original equation developed by Erol and Eksin. In some sense, the weighted average of $X^{\text {gbest }}$ and $X^{c}$, controlled by $\beta$, may be viewed as equivalent to an elitist strategy, wherein the best solution is allowed to influence the direction of the search over many iterations of the technique. Second, for continuous design variables, a multiPhase search is applied to potentially improve the overall search performance. In a two-phase search, the BB-BC algorithm is initially applied to the entire search space and after convergence, a Phase 2 search is conducted in a reduced search space center around $X^{\text {gbest }}$ from Phase 1.

Subsequent Big Bangs and Big Crunches are repeated until the global best solution, has not changed for a number of consecutive iterations; with this condition reached, the BB-BC algorithm is considered to have converged to a solution. At this point, Phase 1 of the BB-BC search is complete. To encourage a refined local search, a Phase 2 BB-BC search is initiated in the region surrounding $X^{\text {gbest }}$. In Phase 2, the search space is redefined around the values encoded in $X^{\text {gbest }}$ from Phase 1 and a fraction of the search space immediately smaller and larger than global best values [35]. Therefore boundary of variables in Phase 2 is defined as

$$
\left\{\begin{array}{l}
X_{\min }=X^{\text {gbest }}-0.5 \times \eta \times(\text { overall search space }) \\
X_{\max }=X^{\text {gbest }}+0.5 \times \eta \times(\text { overall search space })
\end{array}\right.
$$

Which $\eta=$ size of search space in Phase 2 .

Also, at the beginning of Phase 2, the $X^{\text {gbest }}$ solution from Phase 1 can be either maintained or reset. In this paper is assumed the best global solution which obtained from Phase 1, be kept. 


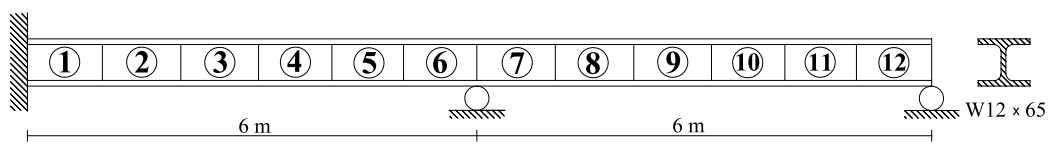

Fig. 2. Geometry of continuous beam.

\subsection{Objective function}

To minimize the objective assessment function, the BB-BC algorithm is used and it attempts for the best solution to a given problem. This objective function is used to provide a measure of how individuals have performed in the problem domain for damage detection. It should be noted that in the case of a minimization problem, the most fitness of candidate solutions will have the lowest numerical value of the associated objective function. In this study, the statement for the objective function is given as

$$
F=1-\operatorname{ECBI}(X)
$$

Where $d=\left\{d_{1}, d_{2}, \ldots, d_{N}\right\}$ are damage parameters at the elements from 1 to $N$.

Using variables of the structure that are sufficiently sensitive to the damage parameters help to avoid ill conditioning problems. Some dynamic properties such as mode shapes and the natural frequencies can be obtained by modal analysis methods. Natural frequencies are easy to measure comparatively and are used in this paper.

\subsection{Numerical examples}

In the present study, damage identification strategy is applied on a continuous beam bridge, the Belgian truss bridge, and a two-story two-bay unbraced frame structure, to illustrate the applicability of the proposed approach.

For the BB-BC algorithm a population of 20 candidates for all examples is selected. Termination criterion for each phase is determined when the value of $X^{\text {gbest }}$ has not improved for the 30 iteration or algorithm reached to 10000 analyses.

The parameters of $\alpha, \beta$ and $\eta$ are considered 1, 0.2 and 0.1 respectively. The algorithm has been applyed in the commercial MATLAB software.

The flowchart of damage detection via Big Bang-Big Crunch algorithm is shown in Fig. 1.

In order to investigate the noise effects on the results of the BB-BC method, the noise is considered for the natural frequencies on complete and incomplete (first ten modes) modal data. The $i$ th noisy response $r_{d_{i} \text { (noisy) }}$, is simulated by [19]:

$$
r_{d_{i}(\text { noisy })}=(1+\sigma \gamma) r_{d_{i}}
$$

Where $\sigma$ is the noise level and $\gamma$ is a random number in the interval $\left[\begin{array}{ll}-1 & 1\end{array}\right]$. In this study $\sigma$ is considered $1.0 \%$.

\subsection{Damage detection in beam bridges}

Damage was detected in beam bridges with specifications as follow:

A two bays continuous beam which contains 12 elements, 13 nodes and 22 nodal DOFs.

The cantilever beam is a $\mathrm{W} 12 \times 65$ with mechanical properties of:

$$
\begin{aligned}
A & =0.0123 \mathrm{~m}^{2}\left(19.1 \mathrm{in}^{2}\right) \\
I & =2.218 \times 10^{-4} \mathrm{~m}^{4}\left(533 \mathrm{in}^{4}\right) \\
E & =207 \times 10^{9} \mathrm{~N} / \mathrm{m}^{2} \\
\rho & =7780 \mathrm{~kg} / \mathrm{m}^{3}
\end{aligned}
$$

A continuous steel beam is shown in Fig. 2. For the reason of modal analyzing the beam was divided in to 12 (two-dimensional) beam elements, 6 element in each bay. 


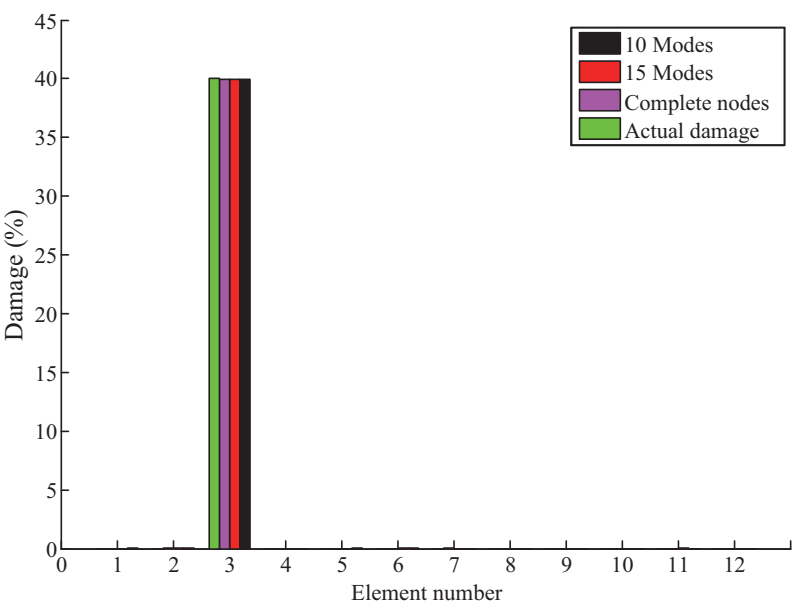

(a) Without noise

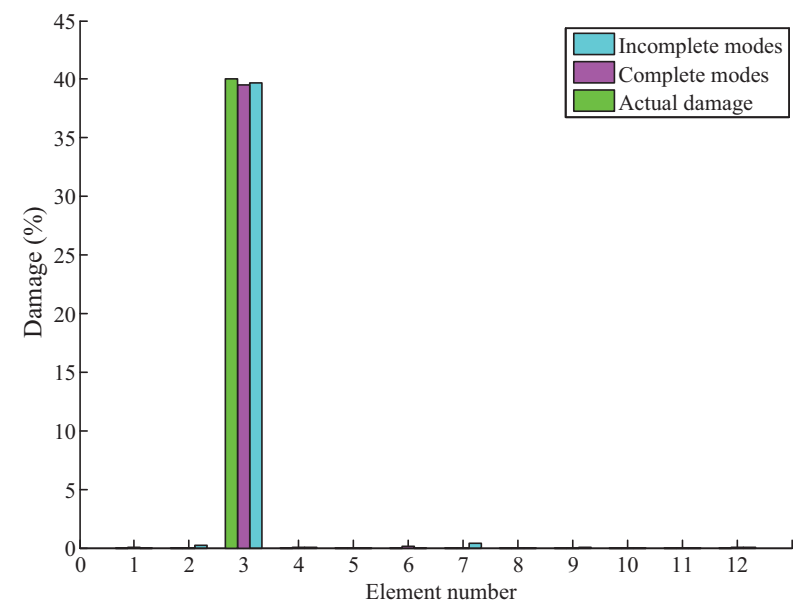

(b) With noise

Fig. 3. Damage distribution of continuous beam using the complete and incomplete modal data in Scenario 1.

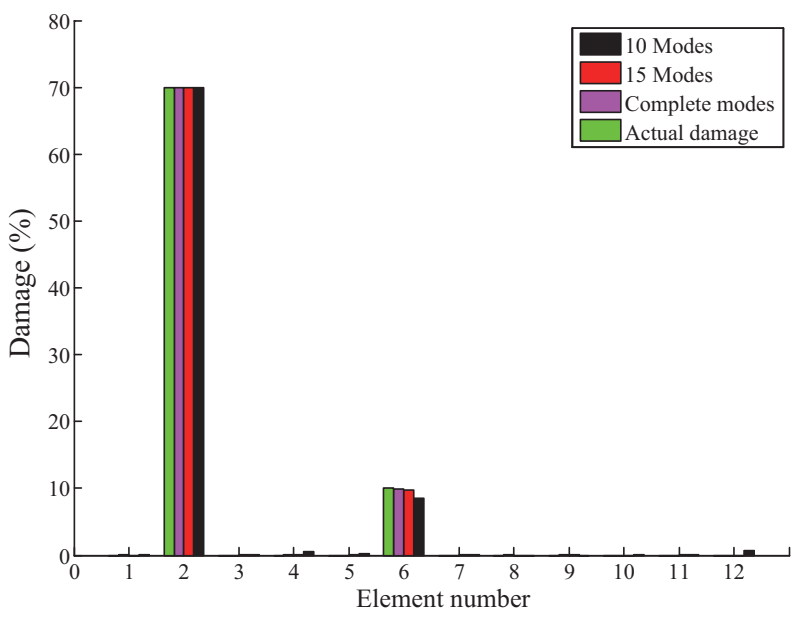

(a) Without noise

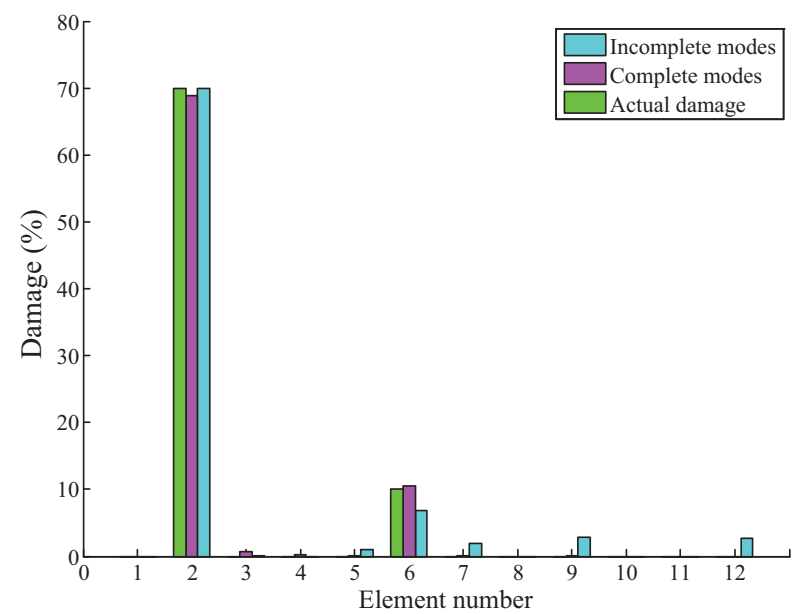

(b) With noise

Fig. 4. Damage distribution of continuous beam using the complete and incomplete modal data in Scenario 2.

For the beam damage detection, three different simulated damage scenarios are considered:

Scenario (1): The stiffness of element 3 was reduced by 40 percent.

Scenario (2): The stiffness of elements 2 and 6 were reduced by 70 and 10 percent, respectively.

Scenario (3): The stiffness of elements 1, 5, 9 and 12 were reduced by 60, 20, 40 and 10 percent, respectively.

These damage scenarios are considered to find the effect of severity of damage (stiffness reduction), number of damaged elements and contribution of damage elements on the results.

In inverse procedure instead of experimental measurements, numerically generated measurements were used. Because of the number of locations at which the modal data is measured from the test is usually much smaller than the degrees of freedom in the numerical model that defines the stiffness and mass matrices and only the lowest frequencies are experimentally measured, the effect of incomplete data in the accuracy of the results will be study.

Diagrams of the BB-BC algorithm's damage detection for beam with complete data (22 modes) and incomplete data (10 and 15 modes for no noisy data and 10 modes for noisy data) is plotted in Figs 3-5.

The figures show this method is able to detect the location and magnitude of damaged elements in all scenarios using complete and incomplete data. It can be observed that, when noise is taken into account in the damage detection problem, the BB-BC method can detect the damage locations and extent close to the correct. 


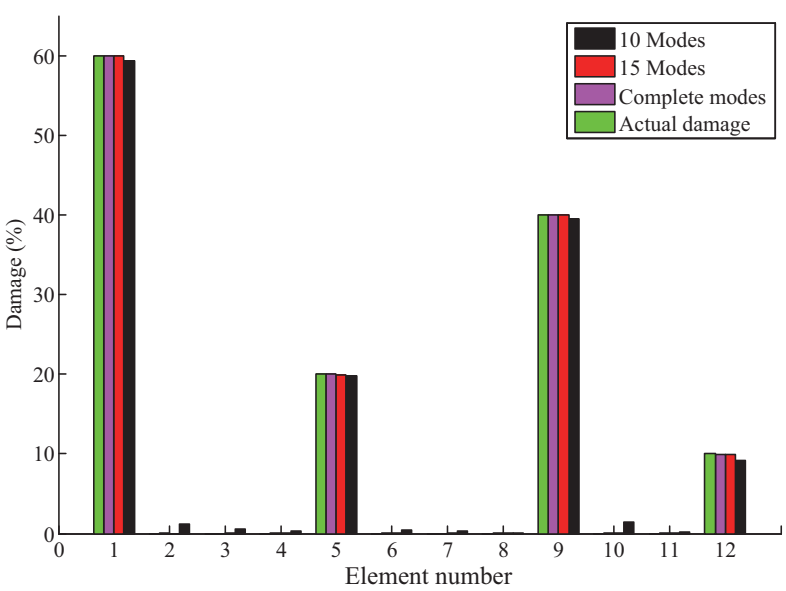

(a) Without noise

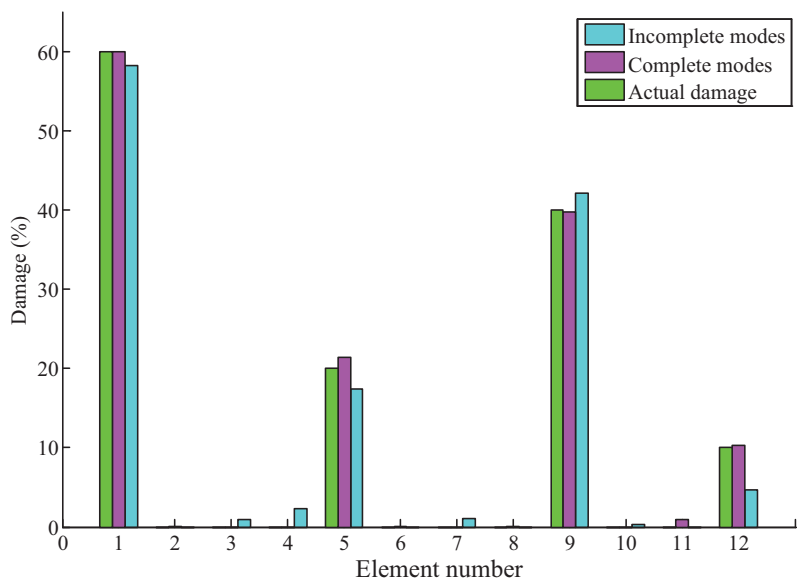

(b) With noise

Fig. 5. Damage distribution of continuous beam using the complete and incomplete modal data in Scenario 3.

Table 1

\begin{tabular}{lc} 
Cross-sectional areas of truss elements \\
\hline Member & Area $\left(\mathrm{cm}^{2}\right)$ \\
\hline $1-6$ & 18 \\
$7-12$ & 15 \\
$13-17$ & 10 \\
$18-21$ & 12
\end{tabular}

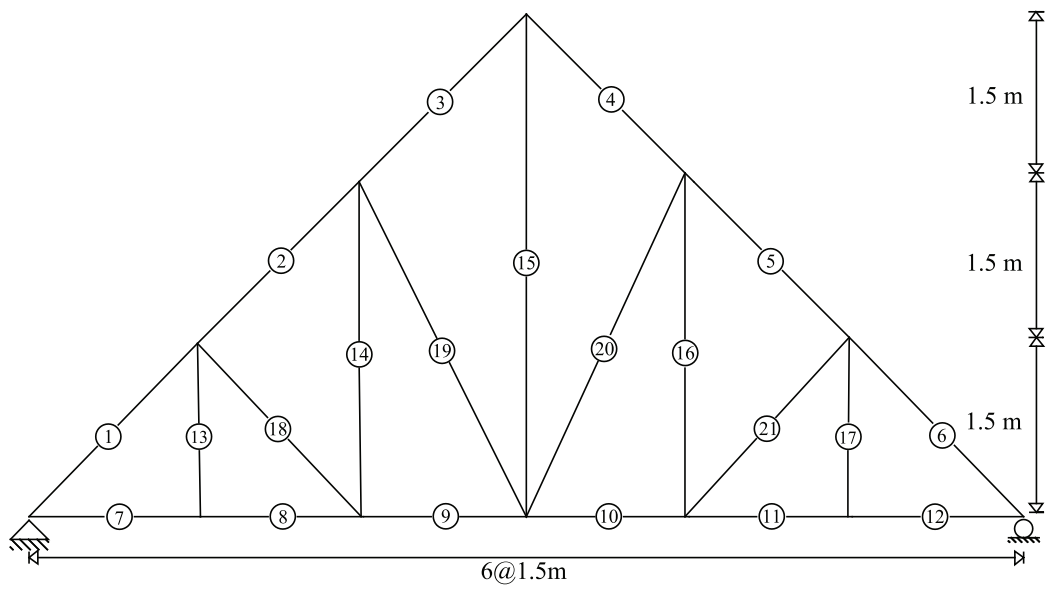

Fig. 6. Geometry of Belgian truss.

\section{Belgian truss bridge}

Geometry of a two-dimensional Belgian truss bridge is shown in Fig. 6. A Belgian truss which contains 21 truss elements, 12 nodes and 21 nodal DOFs. It is used to evaluate the ability of the current damage detection method. The material constants are listed below:

$$
\begin{aligned}
E & =207 \times 10^{9} \mathrm{~N} / \mathrm{m}^{2} \\
\rho & =7780 \mathrm{~kg} / \mathrm{m}^{3}
\end{aligned}
$$

Cross-sectional areas of truss elements are given in Table 1. Three different damage scenarios were subjected to the Belgian truss. Different locations and severity of damage for scenarios are investigated to check the method performance:

Scenario (1): A simple damage- the stiffness of element 15 was reduced by 90 percent.

Scenario (2): Multiple damage- the stiffness of elements 2 and 11 were reduced by 30 and 50 percent respectively.

Scenario (3): Multiple damage- the stiffness of elements 4, 13 and 20 were reduced by 10,40 and 70 percent respectively.

Diagrams of the BB-BC algorithm's damage detection with complete (21 modes) and incomplete dynamic noisy data is plotted in Figs 7-9. 


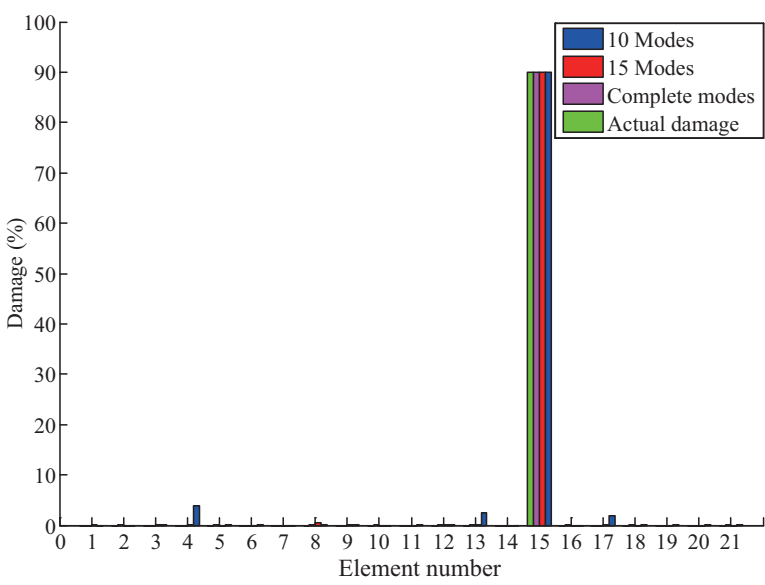

(a) Without noise

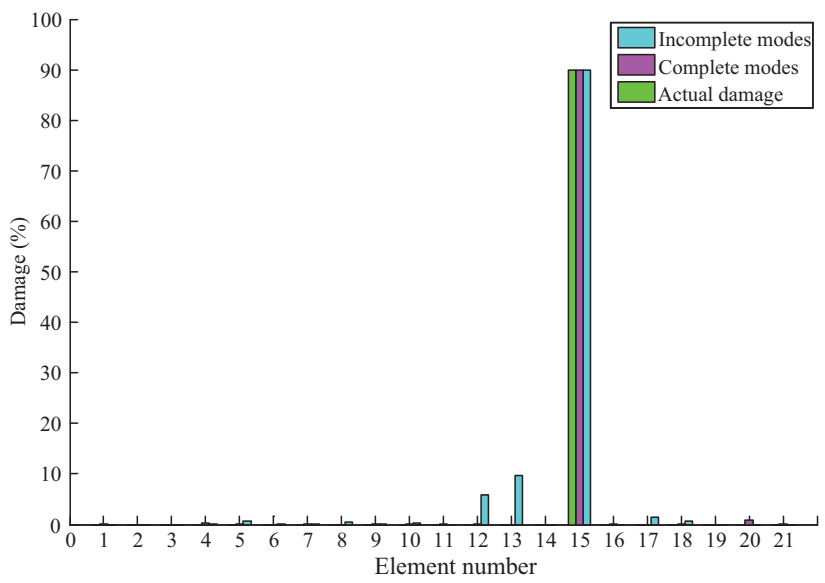

(b) With noise

Fig. 7. Damage distribution of Belgian truss using the complete and incomplete modal data in Scenario 1.

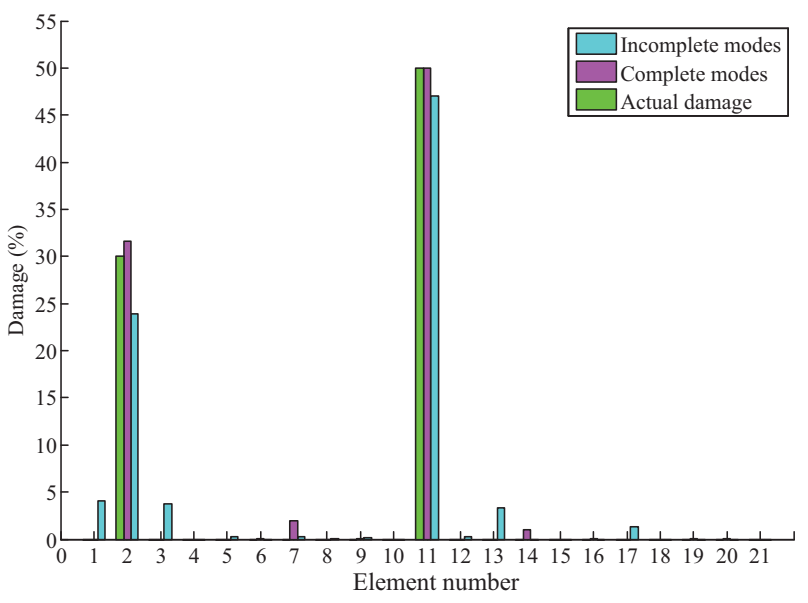

(a) Without noise

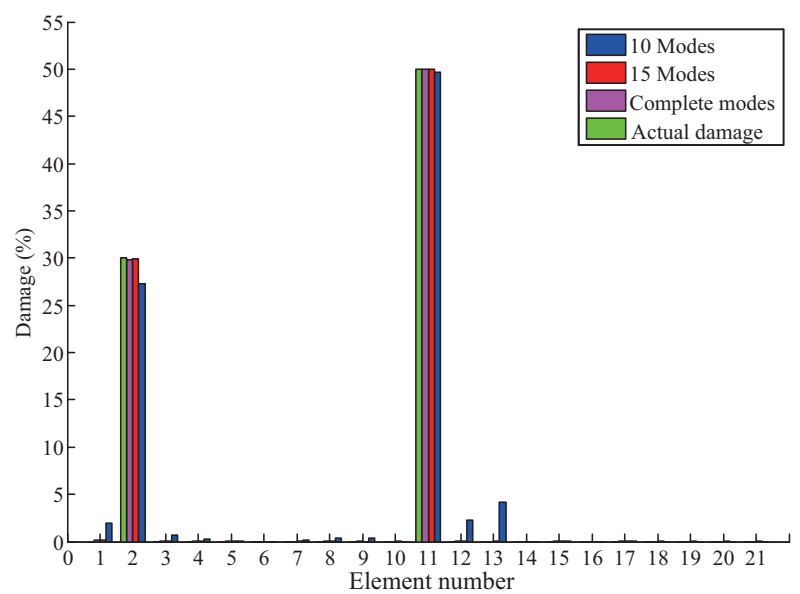

(b) With noise

Fig. 8. Damage distribution of Belgian truss using the complete and incomplete modal data in Scenario 2.

From the results it is clear that if the responses of the structure to be without noise, the search algorithm for damage detection lead to results with high accuracy in all three scenarios. Even in the case where only first ten modes are considered, answers are only slightly less accurate.

\subsection{Two story two-bay unbraced frame structure}

A two-story two-bay frame as shown in Fig. 10 is used to verify the damage detection method explained in this paper. Two-story two-bay unbraced frame structure with 24 elements, 23 nodes and 60 nodal DOFs.

For the unbraced plane frame problem, all columns W14 $\times 132\left(A=0.025 \mathrm{~m}^{2}\left(38.8 \mathrm{in}^{2}\right), I=6.386 \times 10^{-4} \mathrm{~m}^{4}\right.$ $\left(1530 \mathrm{in}^{4}\right)$ ) and all beams W12 $\times 65$. The Young's modulus of steel is $E=207 \times 10^{9} \mathrm{~N} / \mathrm{m}^{2}$, Poisson's ratio is $\nu=0.3$ and the mass density is $\rho=7780 \mathrm{~kg} / \mathrm{m}^{3}$.

Three scenarios are applied in this example:

Scenario (1): Multiple damage- the stiffness of elements 14 and 15 were reduced by 30 percent.

Scenario (2): Multiple damage- the stiffness of elements 4, 6, and 13 were reduced by 70, 10, and 50 percent, respectively. 


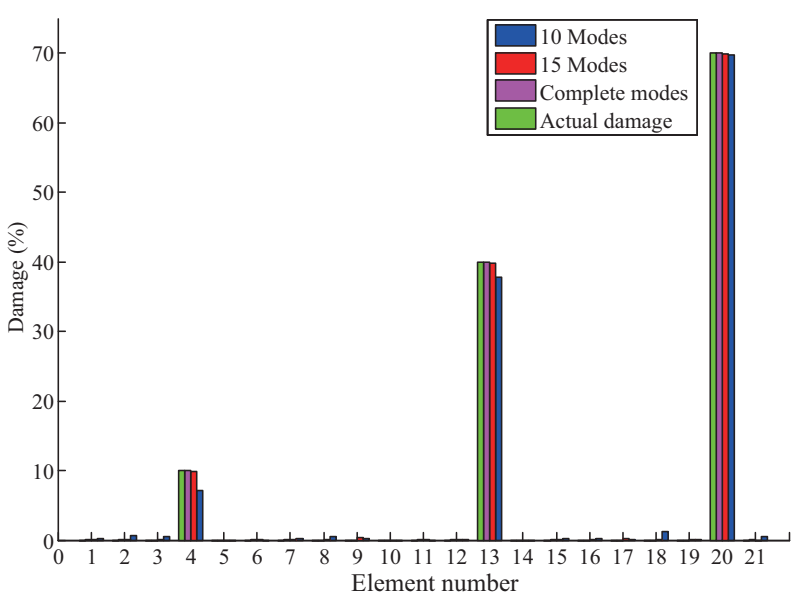

(a) Without noise

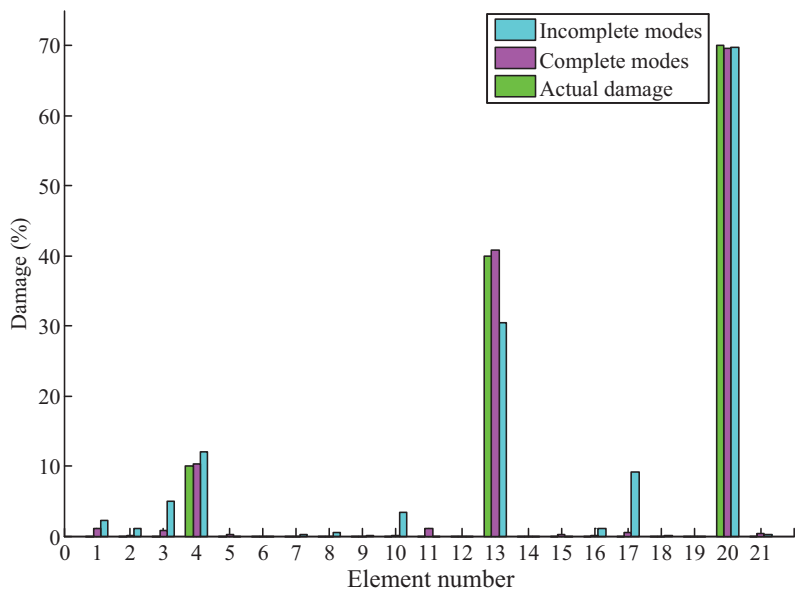

(b) With noise

Fig. 9. Damage distribution of Belgian truss using the complete and incomplete modal data in Scenario 3.

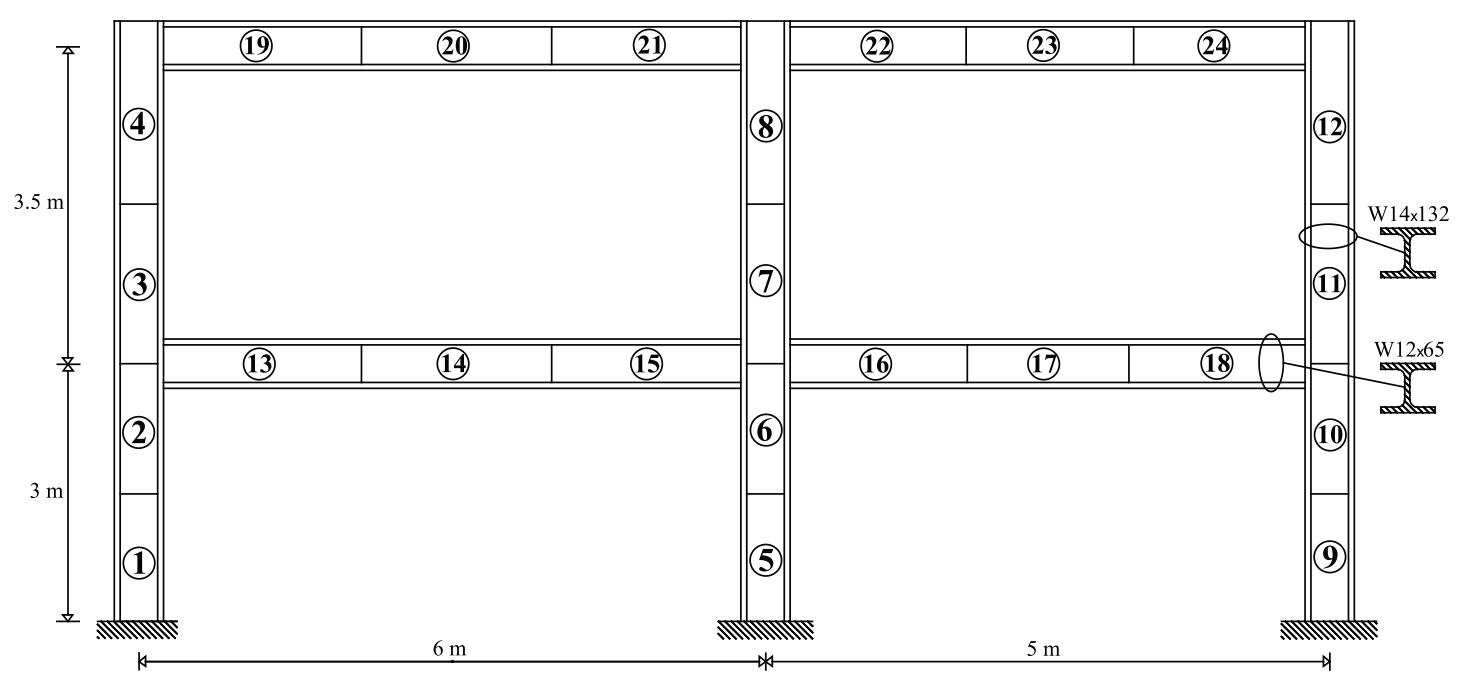

Fig. 10. Geometry of unbraced plane frame.

Scenario (3): A large number of damage- the stiffness of elements 2, 5, 6, 10, 15, 19, 21 and 24 were reduced by $70,30,40,10,20,60,50$ and 80 percent, respectively.

Two cases of complete case with 60 modes and incomplete case is considered which in incomplete case, 20 first modes was considered in damage detection for first and second scenarios, and 45 first modes for last scenario.

The results under three scenarios for unbraced plane frame are shown in Figs 11-13.

Bar charts for the first two scenarios show the significant accuracy even with the one-third of the total number of modes.

The diagram of last scenario indicates the high power of this algorithm in detecting place and severity of damage with considering of a large number of damage (one-third of total elements) and different severities.

\subsection{Comparison of the results with particle swarm optimizer with passive congregation algorithm}

For compare of the presented method with one other method, the performance of the BB-BC algorithm in damage detection is performed using the particle swarm optimizer with passive congregation algorithm (PSOPC) [40]. 


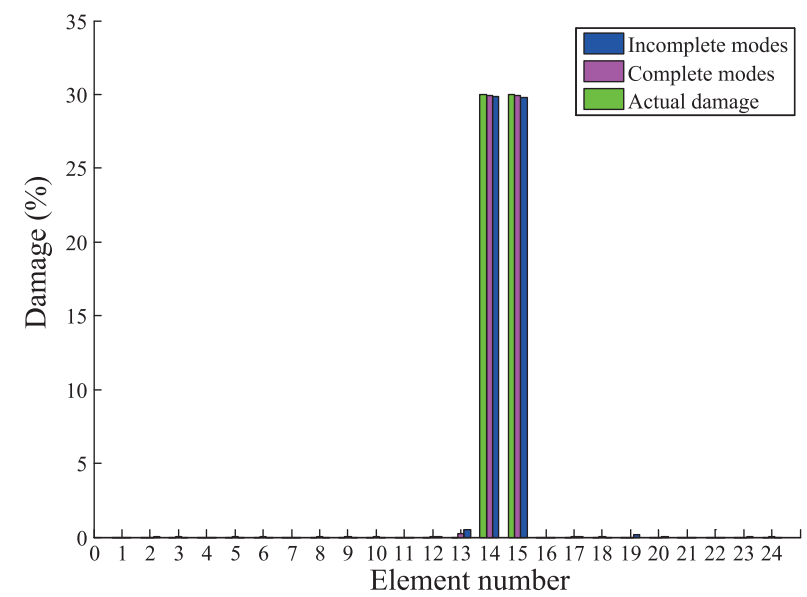

Fig. 11. Damage detection via BB-BC for two damage locations of frame (Scenario 1).

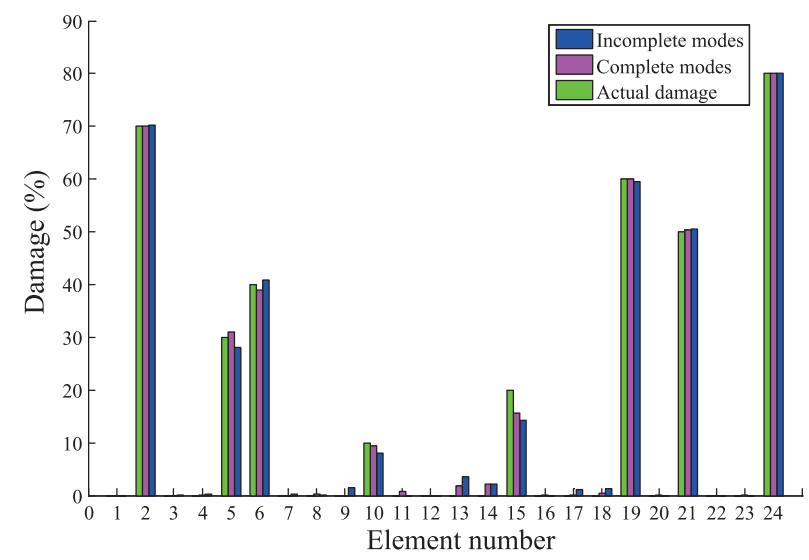

Fig. 13. Damage detection via BB-BC for a large number of damage locations of frame (Scenario 3).

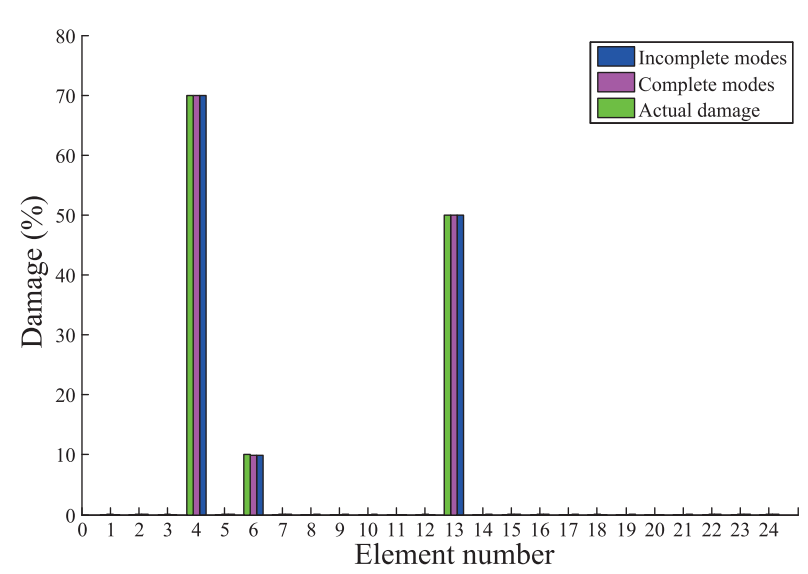

Fig. 12. Damage detection via BB-BC for three damage locations of frame (Scenario 2).

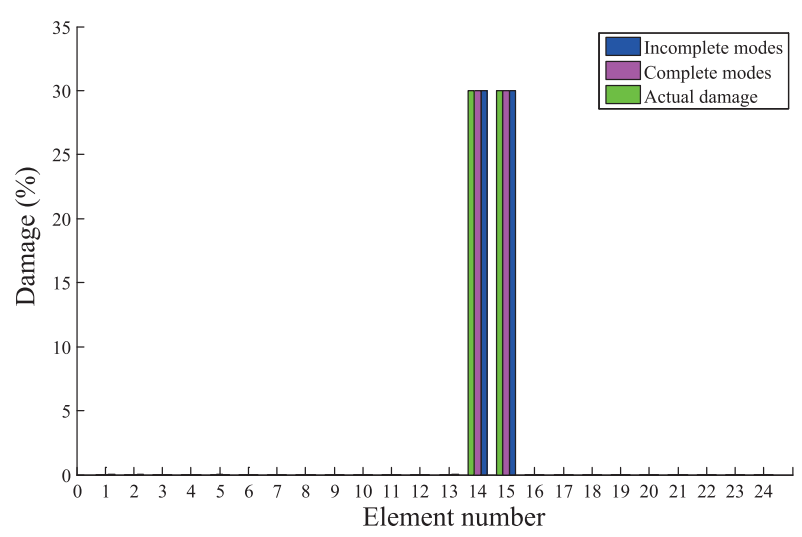

Fig. 14. Damage detection via PSOPC for two damage locations of frame (Scenario 1).

PSOPC algorithm is a particle swarm optimization method with passive congregation's capacity. Adding the passive congregation model to the PSO may increase its performance [41].

In this study, termination criterion is considered when the value of $X^{\text {gbest }}$ has not improved for the 60 iterations or algorithm is reached to the number of 20000 analyses.

Best results from 30 independent runs of PSOPC are selected and are shown in Figs 14-16.

Comparing of first two scenarios in PSOPC and BB-BC algorithm with low number of damages shows the satisfactory damage detection. Third scenario with large number of damages shows different results in two algorithms. As is shown in Figs 13 and 16, place and severity of damage is detected via BB-BC algorithm with higher accuracy rather than the PSOPC. It should be noted that PSOPC in some elements is detected wrong damages in safe elements. In addition, the obtained amount of damage for some of the injured members, has been difference with the actual value.

In order to more accurate comparing the mentioned methods, a new parameter called "average error" is defined Eq. (15) that for any two algorithms are demonstrated in Table 2.

$$
\text { Average error }=\frac{\sum_{i=1}^{N}|d(i)-D(i)|}{N}
$$


Table 2

The compare of average errors of BB-BC and PSOPC for two-story two-bay frame structure

\begin{tabular}{|c|c|c|c|c|c|c|}
\hline & \multicolumn{2}{|c|}{ Scenario1 } & \multicolumn{2}{|c|}{ Scenario2 } & \multicolumn{2}{|c|}{ Scenario3 } \\
\hline & Complete & Incomplete & Complete & Incomplete & Complete & Incomplete \\
\hline BB-BC & 0.000209 & 0.000439 & 0.000177 & 0.000201 & 0.00529 & 0.009021 \\
\hline PSOPC & 0.000005 & 0.000059 & 0.000001 & 0.000001 & 0.06038 & 0.098852 \\
\hline
\end{tabular}

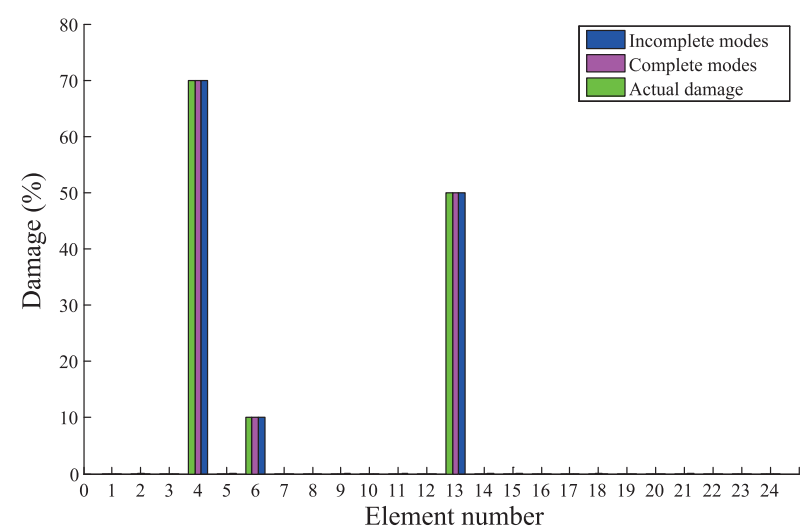

Fig. 15. Damage detection via PSOPC for three damage locations of frame (Scenario 2).

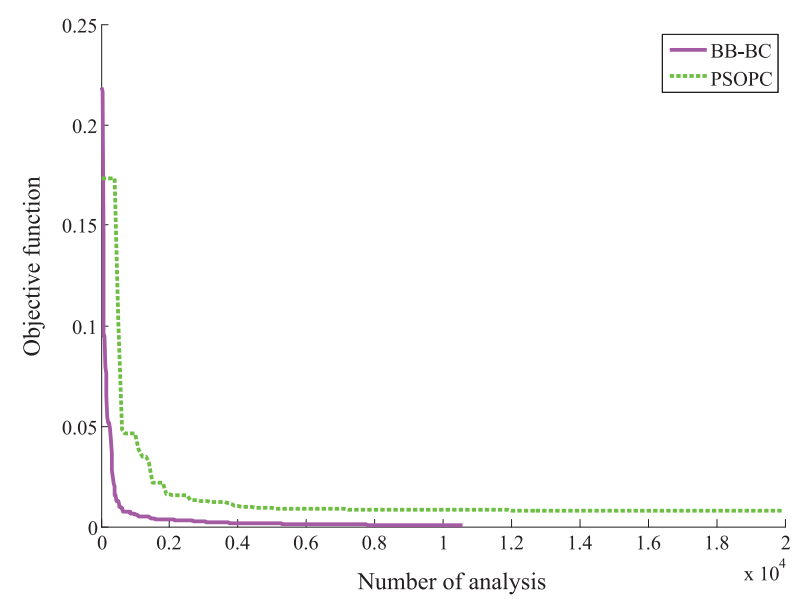

(a) Complete modes

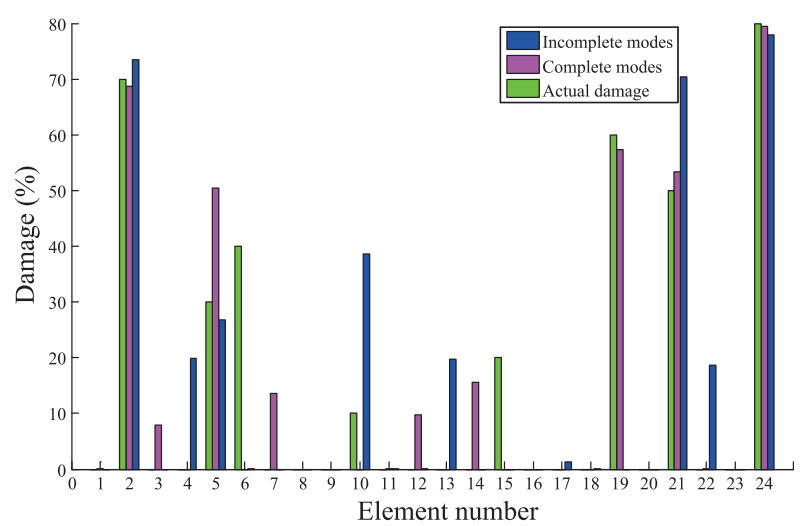

Fig. 16. Damage detection via PSOPC for a large number of damage locations of frame (Scenario 3).

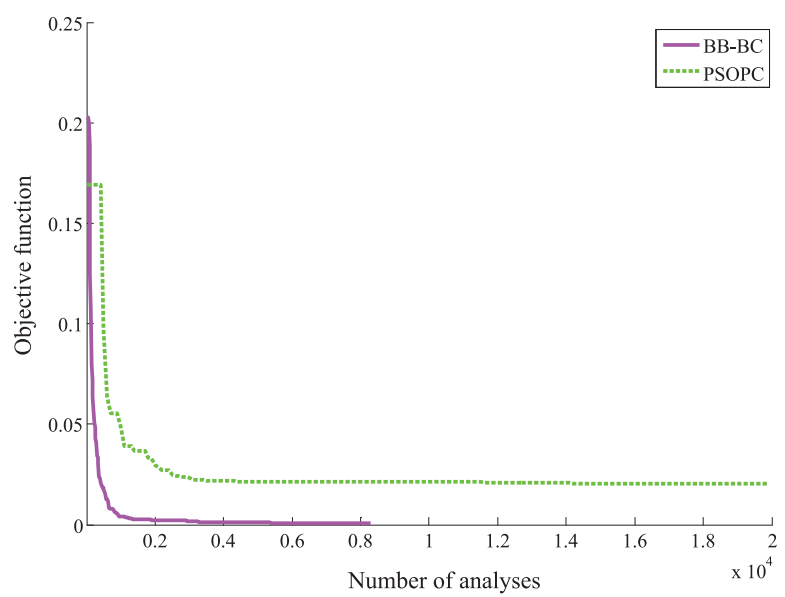

(a) Incomplete modes

Fig. 17. The convergence history for the two-story two-bay frame structure in Scenario 3.

Which $d(i)$ and $D(i)$ are predicted and actual damage in $i$ th element of structure.

From comparison of average errors, it is concluded that in first two scenarios, the accuracy of both methods were suitable; While the errors are close to zero, however the errors of PSOPC algorithm was less than BB-BC algorithm.

In thired scenario with a large number of damage, the mean errors of PSOPC on complete and incomplete modes is greater than BB-BC, while for BB-BC this value is acceptable.

Figure 17 provides the comparison of convergence rates of the PSOPC and BB-BC algorithms for two-story two-bay frame structure in Scenario 3 on complete and incomplete modal data.

From Fig. 17, it can be observed that the PSOPC algorithm cannot find a good result (considering objective function) while the BB-BC algorithm can find the best result. PSOPC algorithm achieve optimal results after 20,000 analyses, However, BB-BC needs 10,600 and 8300 analyses to reach a good solutions in complete and incomplete modal data, respectively. 


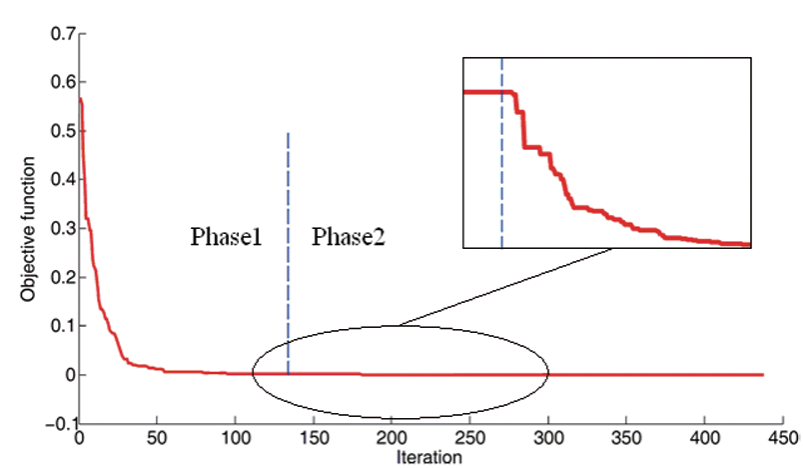

Fig. 18. Converge history of Belgian truss.

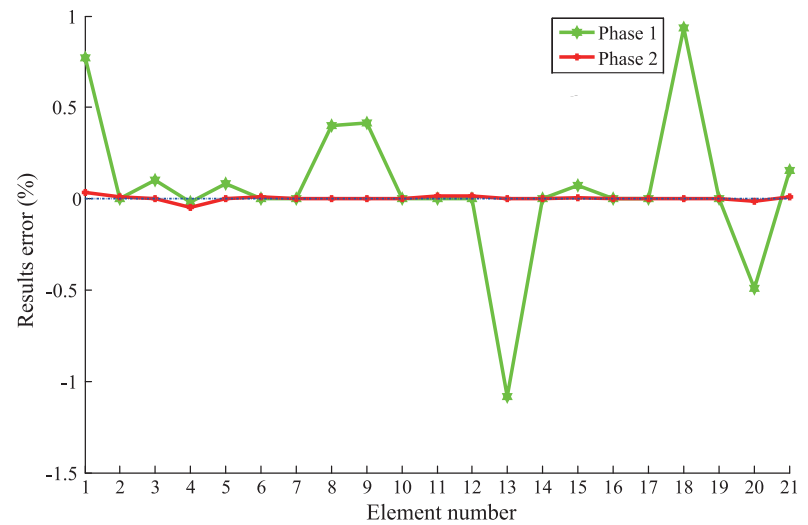

Fig. 19. The results error of BB-BC in any phases for Belgian truss in Scenario 3.

\subsection{The performance of phases of the $B B-B C$ algorithm}

BB-BC algorithm by two-phase performance in the search space allowed navigates solution vector for minimizing the objective function. When the algorithm stops in the first phase, the global search is ended and the second phase is begun to local search of optimum solution.

The algorithm performance during the two phases to detect damage on the Belgian truss in first scenario is shown in Fig. 18. This figure shows how objective function until reach the stopping criterion at every phase is minimized by successive iterations.

The value of results error obtained for each variable of the solution vector comparing with the exact values for the each phase is shown in Fig. 19.

As shown in Fig. 18 the objective function in Phase 1 with downward process is coming near to zero. After the convergence in this phase (end of global search), searching in the second phase around of the best solution obtained from Phase 1 is begun. Because of further reduction of the objective function, this proceeding is done. According to Fig. 19, with search in the second phase has reduced the value of errors significantly and causes results of algorithm to be close to actual damages.

\section{Conclusions}

An approach for detecting damage in structural members based on continuum damage model using new and powerful algorithm named Big Bang-Big Crunch (BB-BC) has been presented. The algorithm evaluates the location and severity of damage in a three structure types: a continuous beam, a Belgian truss and a two-story two-bay plane frame by minimizing an objective function by measuring complete and incomplete modal data with and without noise, considering different damage scenarios.

The results show high accuracy the method of damage detection by BB-BC algorithm even in large structures with a lot of damages. The influence of considering the less number of modes on results accuracy was very low, so that the difference in results compared to the exact results even in many damages was little.

The efficiency of the presented method is examined from a comparison between the results of proposed method and particle swarm optimizer with passive congregation algorithm (PSOPC). The comparison between the two algorithms shows that the BB-BC algorithm with low computational cost and high accuracy can identify damage, even in a large number of damage.

The results of the noise effect on the accuracy of answers obtained from the BB-BC algorithm shows that, since due to noise, the real value of natural frequencies of structure will change, the different characteristics of the actual structure are introduced to algorithm, and this causes reduced accuracy results, especially in the case that only a part of dynamic data of structure is available (incomplete modes). It can be seen from the diagrams that in these cases, 
accuracy reduction of the results of the BB-BC algorithm is very low. This indicates the high power of this algorithm in damage detection considering noise in structures.

BB-BC algorithm by two phase's performance in the search space allowed navigates solution vector for minimizing the objective function. Global search is done in the first phase by this algorithm to find the region that the optimum solution exist in this region, then the second Phase is begun around the best answer obtained from first phase. This search in continue until the difference between the damage variables and the exact solution vector variables be small as much as possible.

\section{References}

[1] Y.J. Yan, L. Cheng, Z.Y. Wu and L.H. Yam, Development in vibration-based structural damage detection technique, Mech Syst Signal process 21(5) (2007), 2198-2221.

[2] T.H.T. Chan, L. Yu, H.Y. Tam, Y.Q. Ni, S.Y. Liu, W.H. Chung and L.K. Cheng, Fiber bragg grating sensors for structural health monitoring of Tsing Ma bridge: Background and experimental observation, Eng Struct 28(5) (2006), 648-659.

[3] F.H. Administration, Reliability of visual inspection, Rep Nos FHWA-RD-01-020, Washington, D.C., 2001.

[4] L. Cartz, Non-destructive testing, ASM International, Materials Park, Ohio, 1995.

[5] B. Raj, T. Jayakumar and M. Thavasimuthu, Practical non-destructive testing, 2nd Ed., Narosa Publishing House, New Delhi, India, 2002.

[6] C.H. Jenkins, L. Kjerengtroen and H. Oestensen, Sensitivity of parameter changes in structural damage detection, Shock and Vibration 41 (1997), 27-37.

[7] J.L. Schulz, B. Command, G.G. Goble and D.M. Frangopol, Efficient field testing and load rating of short and medium-span bridges, Struct Eng Rev 73 (1995), 181-194.

[8] S.W. Doebling, C.R. Farrar, M.B. Prime and D.W. Shevitz, Damage identification and health monitoring of structural and mechanical systems from changes in their vibration characteristics: A literature review, Research Report No. LA-13070-MS, ESA-EA, Los Alamos National Laboratory, NM, 1996.

[9] D.J. Ewins, Modal testing: Theory and practice, Wiley, New York, 1984.

[10] S.W. Doebling, C.R. Farrar and M.B. Prime, A summary review of vibration-based damage identification methods, Shock and Vib 30(2) (1998), 91-105.

[11] N. Hu, X. Wang, H. Fukunaga, Z.H. Yao, H.X. Zhang and Z.S. Wu, Damage assessment of structures using modal test data, Int J Solids Struct 38 (2001), 3111-3126.

[12] O.S. Salawu, Detection of structural damage through changes in frequency: A review, Eng Struct 19(9) (1997), 718-723.

[13] N. Bicanic and H. Chen, Damage identification in framed structures using natural frequencies, Int J Numer Methods Eng 40 (1997), 4451-4468.

[14] A.K. Pandey, M. Biswas and M.M. Samman, Damage detection from changes in curvature mode shapes, J Sound and Vib 145(2) (1991), 321-332.

[15] J.E. Mottershead and M.I. Friswell, Model updating in structural dynamics: A survey, J Sound Vib 167(2) (1993), $347-375$.

[16] H. Hao and Y. Xia, Vibration-based damage detection of structures by genetic algorithm, J Comput Civ Eng 16(3) (2002), 222-229.

[17] M.I. Friswell, J.E.T. Penny and S.D. Garvey, A combined genetic and eigensensivity algorithm, Comput Struct 69(5) (1998), 547-556.

[18] R. Perera and R. Torres, Structural damage detection via modal data with genetic algorithms, Journal of Structural Engineering 132(9) (2006), 1491-1501,

[19] S. Gerist, S.S. Naseralavi and E. Salajegheh, Basis pursuit based genetic algorithm for damage identification, Int J Optim Civil Eng 2(2) (2012), 301-319.

[20] L. Yu and Z.Y. Wan, An improved PSO algorithm and its application to structural damage detection, Proceeding of the Fourth International Conference on Natural Computation, IEEE Computer Society, Washington D.C. (2008), 423-427.

[21] L. Yu and P. Xu, Structural health monitoring based on continuous ACO method, Microelectronics Reliability 51(2) (2011), 270-278.

[22] S. Fallahian and S.M. Seyedpoor, A two stage method for structural damage identification using an adaptive neuro-fuzzy inference system and particle Swarm optimization, Asian Journal of Civil Engineering (Building and Housing) 11 (2010), 797-810.

[23] H. Koh and S.J. Dyke, Structural health monitoring for flexible bridge structures using correlation and sensitivity of modal data, Computers \& Structures $\mathbf{8 5}$ (2007), 117-130

[24] R.S. He and S.F. Hwang, Damage detection by a hybrid real parameter Genetic algorithm under the assistance of grey relation analysis, Engineering Applications of Artificial Intelligence 20 (2007), 980-992.

[25] H.Y. Guo and Z.L. Li, Atwo-stage method to identify structural damage sites and extents by using evidence theory and micro-search genetic algorithm, Mechanical Systems and Signal Processing 23 (2009), 769-782.

[26] L. Yu and X. Chen, Bridge damage identification by combining modal flexibility and PSO methods, Prognostics and system health management conference, Macau, 2010.

[27] S.S. Kourehli, G.G. Amiri, M. Ghafory-Ashtiany and A. Bagheri, Structural damage detection based on incomplete modal data using pattern search algorithm, Journal of Vibration and Control (2012), DOI: 10.1177/1077546312438428.

[28] F. Kang, J. Li and Q. Xu, Damage detection based on improved particle swarm optimization using vibration data, Applied Soft Computing 12(8) (2012), 2329-2335.

[29] B. Nanda, D. Maity and D.K. Maiti, Vibration-based structural damage detection technique using particle swarm optimization with incremental swarm size, Int'l J of Aeronautical \& Space Sci 13(3) (2012), 322-329. 
[30] A. Bagheri, H.R. Razeghi and G.G. Amiri, Detection and estimation of damage in structures using imperialist competitive algorithm, Journal of Shock and Vibration 19 (2012), 405-419.

[31] X. Wang, N. Hu, H. Fukunaga and Z.H. Yao, Structural damage identification using static test data and changes in frequencies, Engineering Structures 23 (2001), 610-621.

[32] A. Messina, E.J. Williams and T. Contursi, Structural damage detection by a sensitivity and statistical-based method, Journal of Sound and Vibration 216 (1998), 791-808.

[33] S.W. Doebling, C.R. Farrar, M.B. Prime and D.W. Shevitz, Damage identification and health monitoring of structural and mechanical systems from changes in their vibration characteristics: A literature review, Research Report No. LA-13070-MS, ESA-EA, Los Alamos National Laboratory, NM, 1996.

[34] M. Nobahari and S.M. Seyedpoor, Structural damage detection using an efficient correlation-based index and a modified genetic algorithm, Mathematical and Computer Modelling 53 (2011), 1798-1809.

[35] H.J. Salane and J.W. Baldwin, Identification of modal properties of bridges, J Struct Eng 116(7) (1990), 2008-2021.

[36] S. Salawu and C. Williams, Bridge assessment using forced vibration testing, J Struct Eng 121(2) (1995), 161-173.

[37] J.M. Ndambi, J. Vantomme and K. Harri, Damage assessment in reinforced concrete beams derivatives, Engineering Structures 24 (2002), $501-515$.

[38] O.K. Erol and I. Eksin, New optimization method: Big Bang-Big crunch, Advances in Engineering Software 37 (2006), 106-111.

[39] C.V. Camp, Design of space trusses using Big Bang-Big Crunch optimization, J Struct Eng, ASCE 133 (2007), 999-1008.

[40] S.M.P. Nejad, G.G. Amiri, A. Asadi, E. Afshari and Z. Tabrizian, Damage detection of skeletal structures using particle swarm optimizer with passive congregation (PSOPC) algorithm via incomplete modal data, Accepted for publication, Journal of Computational Methods in Civil Engineering 3(1) (2012), 1-13.

[41] S. He, Q.H. Wu, J.Y. Wen, J.R. Saunders and R.C. Paton, A particle swarm optimizer with passive congregation, BioSystems 78 (2004), 135-147. 

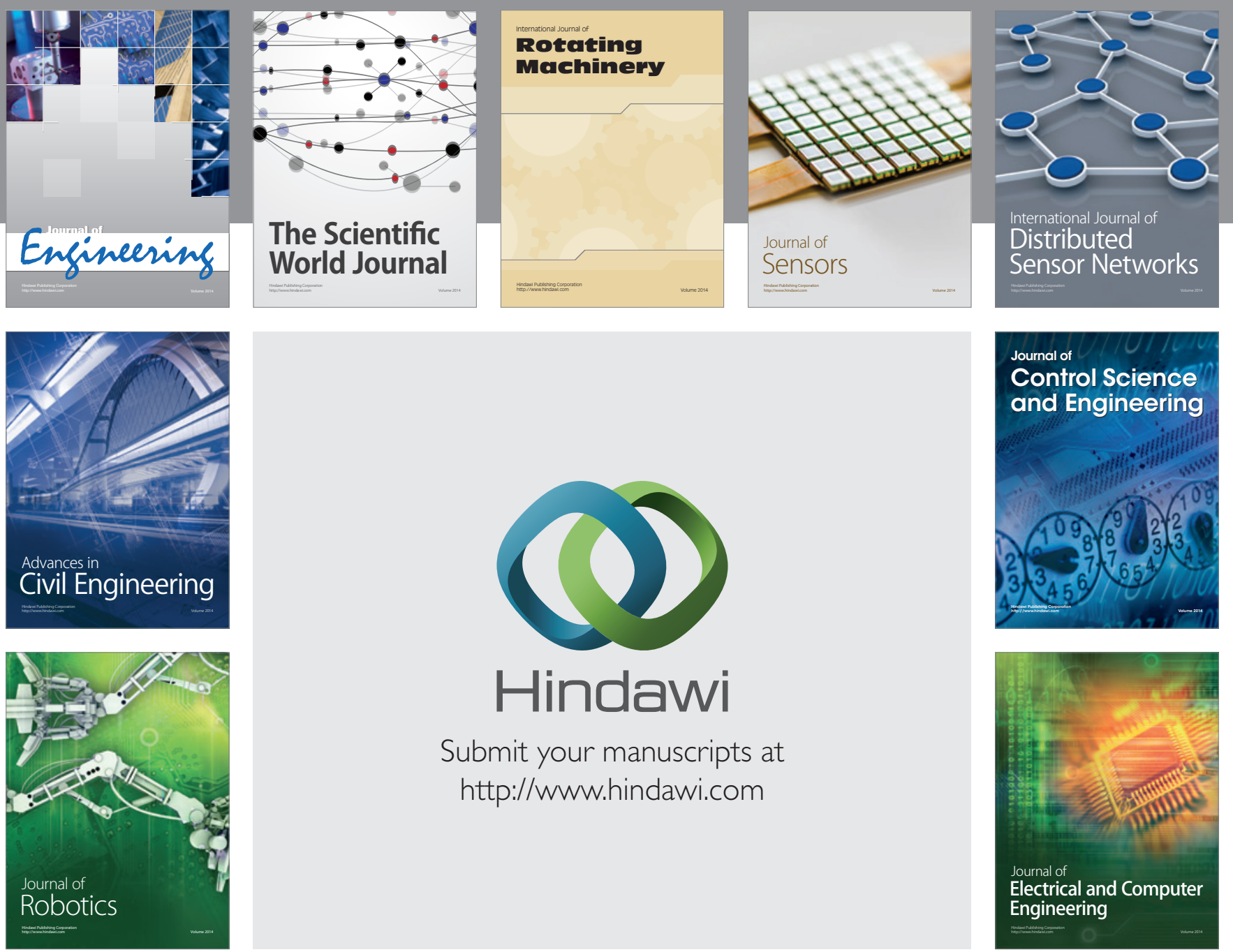

Submit your manuscripts at

http://www.hindawi.com
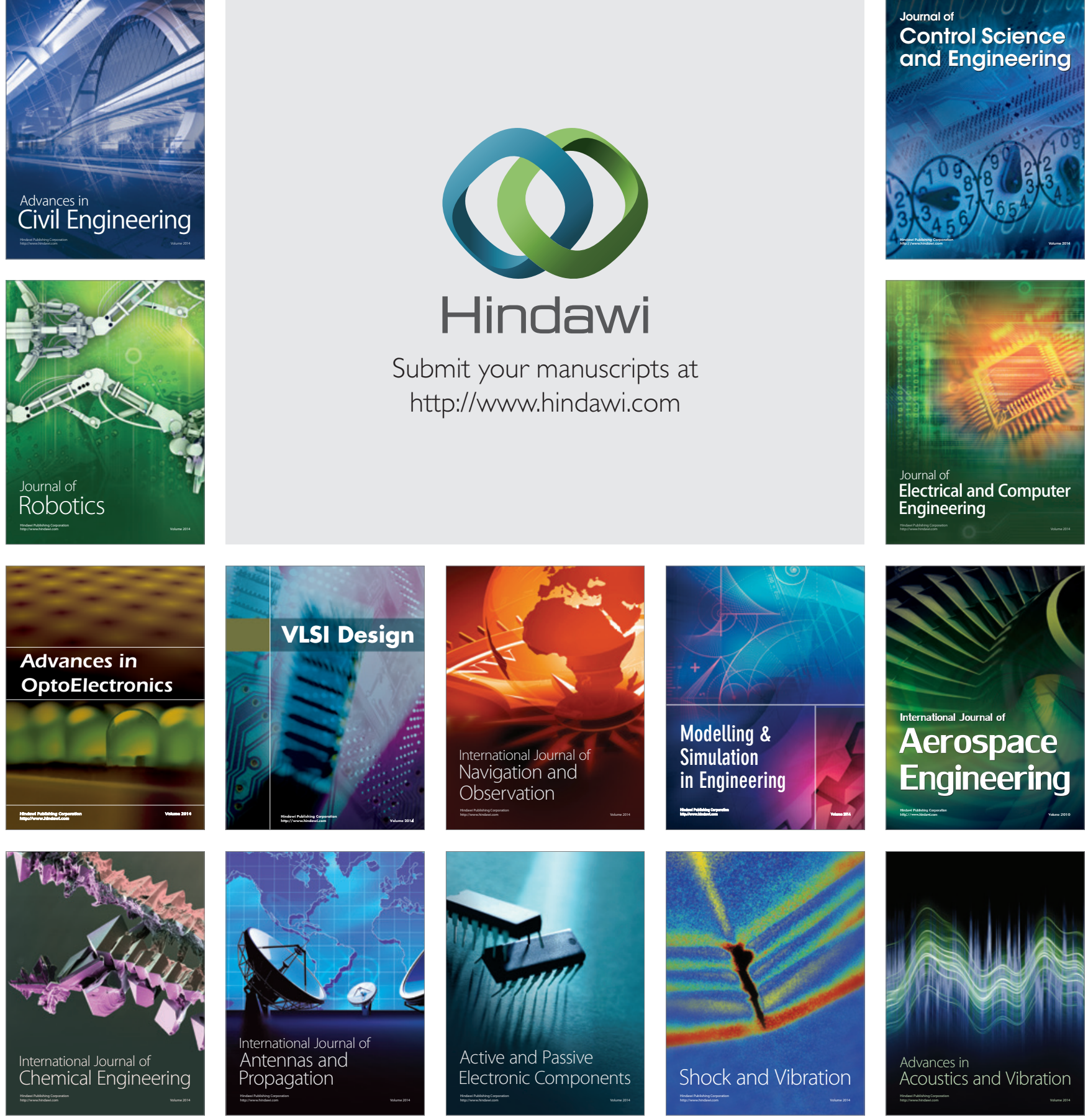